\title{
A PRODUÇÃO CIENTÍFICA BRASILEIRA NO PERIÓDICO SCIENTOMETRICS DE 1978 ATÉ $2006^{1}$ \\ THE BRAZILIAN SCIENTIFIC PRODUCTION IN THE JOURNAL SCIENTOMETRICS FROM 1978 TO 2006
}

\begin{abstract}
Ana Maria Mattos - ammattos@eci.ufmg.br; ammattos@ea.ufrgs.br Mestranda no Programa de Pós-Graduação em Ciência da Informação da Escola de Ciência da Informação da Universidade Federal de Minas Gerais - UFMG Membro do Grupo Pesquisa em Comunicação Científica da Faculdade de Biblioteconomia e Comunicação da UFRGS Ivone Job - ivone.job@ufrgs.br Mestre em Ciência da Informação pelo Programa de Pós-Graduação em Ciência da Informação da Escola de Ciência da Informação da Universidade Federal de Minas Gerais UFMG. Membro do Grupo Pesquisa em Comunicação Científica da Faculdade de Biblioteconomia e Comunicação da UFRGS.
\end{abstract}

\begin{abstract}
Resumo
O objetivo deste artigo é revelar a produção científica dos pesquisadores brasileiros que publicaram artigos na revista Scientometrics, de 1978 até 2006. Classifica os artigos recuperados quanto a tipo de autoria; afiliação e cooperação institucional dos autores; autores mais produtivos; ano de publicação; e número de citações que o artigo recebeu. Os dados analisados revelam: (a) preferência pela autoria compartilhada; (b) predominância de afiliação institucional a universidades e institutos públicos; (c) autores mais produtivos pertencentes às áreas das ciências biológicas e da saúde; (d) predominância de publicação em 1998 e 2006; e (e) cinco artigos que obtiveram entre10 e 25 citações. A pesquisa permite concluir que houve $1,55 \%$ de participação de pesquisadores brasileiros na revista no período analisado; a cooperação institucional internacional predominada pela UFRJ; e que é desejável aumentar a produção internacional de pesquisadores do campo da CI que publicam sobre bibliometria.
\end{abstract}

Palavras-chave: Produção científica. Brasil. Revista científica. Scientometrics.

\section{INTRODUÇÃO}

A implantação do primeiro curso de Mestrado em Ciência da Informação (CI) no Brasil na década de 1970, pelo então Instituto Brasileiro de Bibliografia e Documentação, atual Instituto Brasileiro de Informação em Ciência e Tecnologia (IBICT) provocou no país uma "[...] febre pela bibliometria, sendo esta aplicada aos mais diversos campos" (URBIZAGÁSTEGUI ALVARADO, 1984, p. 92).

Na mesma década, no cenário internacional, destaca-se o ano de 1978 com a publicação do primeiro periódico especializado no assunto, Scientometrics, apontado por pesquisadores respeitados no campo como um dos principais veículos de comunicação científica sobre o tema (SENGUPTA, 1992; MACIAS-CHAPULA, 1998).

A importância deste título se mantém e de acordo com o Portal.periódicos.CAPES ${ }^{2}$, o fator de

\footnotetext{
${ }^{1}$ As autoras agradecem as sugestões de alteração enviadas pelos avaliadores e pela comissão editorial que contribuíram para melhorar este artigo.

${ }^{2}$ http://www.periodicos.capes.gov.br/portugues/index.jsp
} 
impacto atribuído em 2005 a revista Scientometrics pelo Journal Citation Report (JCR) é igual a 1.738. De acordo com Castro (2006, p.131) o fator de impacto é "[...] usado para medir a qualidade das revistas e não trabalhos de autores em particular” e este índice demonstra a qualidade desta revista especializada no assunto.

A Scientometrics possui a característica de ser uma revista preocupada com os aspectos quantitativos da ciência, com a comunicação e política cientificas, com ênfase nas investigações das quais o desenvolvimento e os mecanismos da ciência são estudados por métodos matemáticos e estatísticos. É de caráter interdisciplinar e instrumento indispensável para os administradores de pesquisas, tais como centros de documentação, bibliotecas, agências governamentais científicas e institutos de pesquisas. É editada pela Information Scientific and Scientometrics Research Unit do Instituto de Estudos Políticos da Academia de Ciências da Hungria. (AIMS, 2008). Nela escreveram e escrevem autores renomados da cientometria tais como: Solla Price, Wolfgang Glanzel, Ronald Rousseau, Martin Meyer, Katarina Larsen, Henk F. Moed, dentre outros.

O periódico Scientometrics encontra-se indexado em várias bases inclusive na Social Sciences Citation Index (SSCI), um dos subprodutos mais conhecidos das bases da Web of Science $e$ específica para as ciências sociais e aplicadas. Ela foi criada em 1956 incluindo, atualmente, 1.986 títulos de periódicos sendo 16 deles latino-americanos ${ }^{3}$. Sob a perspectiva dos países, Dutt, Carg e Bali (2003) analisaram 1.317 artigos publicados no periódico Scientometrics de 1978 até 2001 e neste período o Brasil contribuiu com 19 artigos.

Considerando-se a Bibliometria como uma disciplina seminal na introdução e desenvolvimento das pesquisas em CI no Brasil e a ocorrência quase simultânea de um periódico internacional especializado sobre o tema, apresenta-se a seguinte hipótese de pesquisa: dado o estímulo e a oportunidade, ocorreu a visibilidade internacional da produção científica brasileira utilizando a Bibliometria como teoria ou método através da publicação destas pesquisas neste periódico específico?

Dessa forma, o problema desta pesquisa consiste em verificar a visibilidade em âmbito internacional da produção científica dos pesquisadores brasileiros, ligados à CI, que publicaram seus artigos na revista científica Scientometrics ${ }^{4}$ de 1978 até 2006, analisando as seguintes variáveis: (i) o tipo de autoria - se única ou compartilhada; (ii) a ocorrência ou não de cooperação institucional; (iii) a afiliação institucional dos autores; (iv) os autores mais produtivos; (v) o ano da publicação; e (vi) a freqüência em que os artigos foram citados. Interessa particularmente a esse estudo, os pesquisadores brasileiros que investigaram sobre bibliometria ou a utilizaram como método e publicaram os resultados de suas pesquisas em artigos da revista Scientometrics no período de 1978 até 2006.

\section{REFERENCIAL TEÓRICO}

Estudos constataram o Brasil como o país que mais produtivo e mais citado entre os países da América Latina e o segundo mais citado quando considerados os países ibero-americanos, destacando-se principalmente no campo da saúde, química e engenharia elétrica (CARG, 2003). Alguns estudos confirmam esta tendência de ponta também na produção intelectual

\footnotetext{
${ }^{3}$ http://espacio.bvsalud.org/boletim.php?articleId=07171815200749. Acesso em: 24 jul. 2007.

4 Título completo do periódico: Scientometrics: an International Journal for all Quantitative Aspects of the Science of Science, Communication in Science and Science Policy.
}

Enc. Bibli: R. Eletr. Bibliotecon. Ci. Inf., Florianópolis, n. 26, $2^{\circ}$ sem.2008 
brasileira no campo da CI. (ARENAS et al., 2000; MOYA-ANEGÓN; HERREROSOLANA, 2002; HERRERO-SOLANA; RÍOS-GÓMEZ, 2006).

Entretanto, apesar do bom desempenho apresentado pelo Brasil apontado por estes estudos, não se sabe quem são os pesquisadores, a que instituições estão afiliados, que temas estão pesquisando, em que periódicos estão publicados seus artigos e nem quão citada foi essa produção científica.

A primeira dissertação brasileira relacionada à Bibliometria foi defendida por Rosali Pacheco Fernandez em 1973 na Universidade Federal do Rio de Janeiro/IBICT que analisou a produção científica dos grupos de pesquisa sobre física no estado sólido na América Latina. A esta se seguiram outras de caráter bibliométrico. Destaca-se a dissertação que primeiro usou o termo cienciometria na literatura científica na área da Biblioteconomia e CI no Brasil, em 1984, de Regina Célia Montenegro de Lima que analisou as citações no periódico Scientometrics (STUMPF et al., 2006).

A realização de pesquisas científicas e sua comunicação em revistas constituem-se, na atualidade, a forma mais utilizada pelos pesquisadores para divulgar e tornar visível seu trabalho. "A visibilidade é uma característica desejável da comunicação científica" (PACKER; MENEGHINI, 2006, p. 237). Sua presença se aplica em todo o processo da comunicação científica, desde a produção da pesquisa, sua publicação em periódicos até a sua presença nas bases de dados. Provavelmente, as revistas mais prestigiosas da área, são as mais procuradas para publicação porque evidencia, em seus critérios de avaliação uma visibilidade maior, assegurando e transmitindo confiança aos autores. Estes critérios passam pela leitura, avaliação, revisão pelos pares, pela presença do título da revista em bases de dados e em índices que determinam o fator de impacto de determinada publicação. Portanto, não basta publicar, é necessário ser visível no mundo científico.

Na área da bibliometria e cienciometria, o periódico Scientometrics exerce este papel de visibilidade na área, e um artigo publicado em suas páginas, provavelmente será uma referência mais prestigiosa do que em periódicos brasileiros, mesmo que nossos pesquisadores tenham que escrever num idioma que não é sua língua materna, mas é a mais universal, caso da língua inglesa. É natural e desejável que os pesquisadores busquem publicar nos periódicos referenciais de sua área temática, que em última análise vai melhorar e premiar o seu trabalho e contribuir para seu prestígio profissional e pessoal (PACKER; MENEGHINI, 2006).

A CI tem se destacado na condução de pesquisas que analisam a produção em revistas científicas por acreditar que elas ofereçam perspectivas de entendimento da história da construção intelectual de áreas específicas. Vários estudos comprovam a importância da CI para análise de indicadores de produção em outros campos. Para ilustrar, podemos citar:

Dois trabalhos realizados em 2006 na Universidade de São Paulo (USP): o de Telma de Carvalho que analisou a produção científica brasileira em odontologia e sua visibilidade nacional e internacional e o de Rogério Mugnaini que estudou os critérios para adequação da avaliação da produção científica brasileira, comparando os critérios do ISI com os do Scientific Electronic Library Online (SciELO).

Na Universidade Federal do Rio Grande do Sul (UFRGS), Samile Vanz, em 2004, e Rosa Mesquita em 2005 analisaram a área da Comunicação; Fátima Maia, a produção e o uso de informação em saúde na área de epidemiologia.

No Programa de Pós-graduação em Ciências da Secretaria da Saúde de São Paulo, Claudete Gomes dos Santos em 2004 defendeu a dissertação intitulada AIDS em revista(s): produção científica no Brasil de 1982-2002.

Enc. Bibli: R. Eletr. Bibliotecon. Ci. Inf., Florianópolis, n. 26, $2^{\circ}$ sem.2008 
Leandro Ferreira de Carvalho defendeu dissertação sobre bibliometria em saúde coletiva através dos periódicos Cadernos de Saúde Pública e Revista de Saúde Pública, na Escola de Saúde Pública do Rio de Janeiro, em 2005.

Na Universidade do Estado de São Paulo (UNESP), foi apresentada em 2005 a dissertação que faz um estudo exploratório do conceito de periódicos científicos brasileiros da área de CI por Lucilene da Silva Messias.

Estes são apenas alguns exemplos de teses e dissertações que demonstram o interesse atual da bibliometria associada às revistas e produção científica, além de ser tema também de muitos artigos das revistas do campo da CI.

Como exemplo de artigos referencia-se o trabalho de Machado (2007) que faz análise cientométrica dos estudos de bibliometria publicados em periódicos das áreas de biblioteconomia e ciência da informação de 1990-2005 no Brasil. A amostra analisada pelo autor compreendeu 21 artigos assinados por 19 autores em cinco periódicos nacionais. Os resultados apontam uma produção assimétrica no período, com predomínio de autoria única e sem grandes produtores em Bibliometria no Brasil, mas observa que a análise de citações vem impulsionando a área.

Um levantamento realizado na revista Ciência da Informação aponta 19 artigos publicados sobre o tema bibliometria e cientometria no período de 1998-2004. Desses há um estudo de Mugnaini e colaboradores (2004) que analisa indicadores de publicação mostrando o aumento expressivo da produção científica brasileira em bibliometria nos últimos 20 anos, assim como sua internacionalização. Concluiu, também, que há uma concentração desses pesquisadores na região sudeste e que se ampliou a parceria de pesquisadores brasileiros com de outros países, principalmente dos EUA, Europa e na América do Sul.

O que parece evidente é que o interesse pela bibliometria cresceu muito desde a década de 1970 até nossos dias, seja pelo aumento dos cursos de pós-graduação no país e, conseqüentemente, mais pesquisadores publicam, seja pelo acréscimo quantitativo e qualitativo das revistas científicas. Este crescimento ocorreu em todas as ciências. Meadows (1999, p. 15) afirma que a comunidade científica no mundo tem crescido de forma a termos hoje à nossa disposição, provavelmente, para um bate-papo "[...] três quartas partes de todos os pesquisadores registrados durante toda a história escrita [...]”.

\section{MÉTODO}

A busca on-line, empreendida neste estudo, é um processo que agrega valor em termos dos procedimentos de seleção e refinamento realizados com base em estratégias de busca inteligentes. Atualmente, pesquisadores têm desenvolvido estudos, combinando metodologias bibliométricas e o uso de bases de dados de grande porte, visando à análise de tendências e o mapeamento do conhecimento.

Apesar de a revista Scientometrics estar disponível no Portal.periódicos.Capes, a busca não pode ser efetuada de forma direta devido a restrição apresentada ao título pelo período de acesso disponível no Portal que é de 2000 até o presente, não contemplando todo o recorte temporal determinado para esta investigação. Então, para identificar os pesquisadores brasileiros que investigaram sobre bibliometria, tanto como teoria quanto como método, e publicaram seus artigos na revista Scientometrics de 1978 até 2006 foi realizada uma busca junto ao Portal.periódicos.Capes mediante pesquisa na base de dados SSCI.

Um fator importante para a escolha da base SSCI é o método de indexação na base de dados, que registra os dados bibliográficos completos de cada documento, incluindo os resumos em Enc. Bibli: R. Eletr. Bibliotecon. Ci. Inf., Florianópolis, n. 26, $2^{\circ}$ sem.2008 
inglês, os endereços dos autores e editores e as referências bibliográficas citadas em cada artigo. A indexação dos dados de afiliação dos autores é essencial para verificar a cooperação científica entre os autores e as instituições (TESTA, 1998).

A estratégia de busca utilizada para identificar os artigos foi pesquisar no SSCI o título Scientometrics, relacionando-o com a identificação do Brasil ou Brazil no campo address, e selecionando previamente o período de 1978 até 2006. De acordo com estes critérios foram recuperadas 34 referências de artigos publicados por autores brasileiros que foram tabuladas em um arquivo no software Microsoft Excel para análise.

\section{RESULTADOS E DISCUSSÃO}

Inicia-se a análise identificando o tipo de autoria dos artigos, se única ou compartilhada, apresentados a seguir na Tabela 1 .

Tabela 1 - Freqüência de autores por artigo

\begin{tabular}{cccc}
\hline $\begin{array}{c}\text { FREQUENCIA DE } \\
\text { AUTORES }\end{array}$ & $\begin{array}{c}\text { FREQUENNCIA DE } \\
\text { ARTIGOS }\end{array}$ & $\mathbf{( \% )}$ & AUTORES ACUMULADOS \\
\hline 5 & 1 & 2,94 & 80 \\
4 & 4 & 11,76 & 75 \\
3 & 10 & 29,41 & 59 \\
2 & 10 & 29,41 & 29 \\
& & 26,48 & 9 \\
\hline TOTAL & 9 & $\mathbf{1 0 0}$ & $\mathbf{8 0}$ \\
\hline
\end{tabular}

No total 80 autores assinaram os 34 artigos recuperados. Percebe-se uma preferência pela autoria compartilhada em detrimento dos extremos, ficando em média, 2 a 3 autores por artigo (29,41\% da amostra cada um). Mesmo assim, a autoria única representa 26,48\% da amostra. Artigos com cinco autores representam 2,94\% do total, quatro autores dividiram a autoria de quatro artigos, ou 11, 76\%. É importante salientar que 25 artigos do universo de 34 são compartilhados em sua autoria.

A seguir serão analisadas estas parcerias, identificando se são realizadas dentro da mesma instituição dos autores ou fora. Há uma tendência, atualmente, nas ciências sociais e da saúde de compartilhamento de autorias, por diversos motivos, seja por viver-se numa era da interdisciplinaridade ou até por exigências de caráter econômico das fontes financiadoras de pesquisas.

Assim analisa-se a afiliação institucional dos autores e a cooperação institucional, se ocorrerem, para publicação dos artigos. Inicialmente destacam-se os artigos em que não houve parcerias. São os nove artigos de autoria única, em que os pesquisadores são ligados a órgãos nacionais de fomento a pesquisa e formação de pessoal de nível superior como o IBICT, o Conselho Nacional de Desenvolvimento Científico e Tecnológico (CNPq) e a Empresa Brasileira de Pesquisa Agropecuária (Embrapa).

Há também três universidades públicas: USP, Universidade Federal de São Carlos (UFSCar) e Universidade Estadual de Campinas (Unicamp) como pode ser visualizado no Quadro 1. 


\begin{tabular}{|l|r|}
\hline INSTITUIÇÃO & ANO \\
\hline BICT & 1985 \\
\hline CNPq & 1986 \\
\hline CNPq & 1987 \\
\hline Instituto de Química da USP & 1992 \\
\hline Instituto de Química da USP & 1996 \\
\hline Embrapa & 1998 \\
\hline Unicamp & 1999 \\
\hline Departamento de Engenharia de Materiais da UFSCar & 2002 \\
\hline Departamento de Engenharia de Materiais da UFSCar & 2006 \\
\hline
\end{tabular}

Quadro 1 - Afiliação institucional das autorias únicas

A seguir analisam-se os 25 artigos em que ocorreu o compartilhamento entre os autores, não necessariamente entre instituições. No Quadro 2 visualizam-se informações sobre as instituições a que pertencem os autores desses artigos, que representam 73,52\% da amostra, e onde se percebe que a colaboração para publicação, em sua maioria, é entre autores de instituições brasileiras.

\begin{tabular}{|c|c|c|c|}
\hline \multicolumn{2}{|c|}{ FREQÜÊNCIA } & \multirow{2}{*}{ INSTITUIÇÃO } & \multirow{2}{*}{ ANO } \\
\hline AUTOR & ARTIGOS & & \\
\hline 5 & 1 & $\begin{array}{l}\text { Núcleo de Pesquisas sobre Ensino Superior (2) } \\
\text { Núcleo de Pesquisa em Relações Internacionais (2) } \\
\text { Instituto de Matemática e Estatística, todos ligados à USP (1) }\end{array}$ & 2003 \\
\hline \multirow{4}{*}{4} & \multirow{4}{*}{4} & $\begin{array}{l}\text { Instituto de Ciências Biológicas da UFRJ (2) } \\
\text { Departamento de Bioquímica da UFRGS (2) }\end{array}$ & 1997 \\
\hline & & $\begin{array}{l}\text { Instituto de Ciências Biológicas da UFRJ (2) } \\
\text { Departamento de Bioquímica da UFRGS (2) }\end{array}$ & 1998 \\
\hline & & $\begin{array}{l}\text { Instituto de Ciências Biomédicas UFRJ (2) } \\
\text { Departamento de Psiquiatria e Medicina Legal UFRJ (2) }\end{array}$ & 2001 \\
\hline & & Faculdade de Filosofia, Ciências e Letras de Ribeirão Preto da USP (4) & 2006 \\
\hline \multirow{11}{*}{3} & \multirow{11}{*}{10} & $\begin{array}{l}\text { Embrapa (1) } \\
\text { Departamento de Estatística da UnB (2) }\end{array}$ & 1993 \\
\hline & & Embrapa (3) & 1999 \\
\hline & & $\begin{array}{l}\text { Instituto Dante Pazzanese de Cardiologia de São Paulo (1) } \\
\text { Faculdade de Economia, Administração e Contabilidade da USP (1) } \\
\text { Instituto de Saúde de São Paulo (1) }\end{array}$ & 2000 \\
\hline & & $\begin{array}{l}\text { Faculdade de Medicina UFRJ (2) } \\
\text { Instituto de Ciências Biomédicas UFRJ (1) }\end{array}$ & 2003 \\
\hline & & Instituto de Ciências Biomédicas da UFRJ (3) & 2004 \\
\hline & & $\begin{array}{l}\text { Instituto de Bioquímica Médica da UFRJ (1) } \\
\text { Instituto Dante Pazzanese de Cardiologia de São Paulo (1) } \\
\text { Instituto de Química da USP (1) }\end{array}$ & 2005 \\
\hline & & Instituto de Ciências Biológicas da UFG (3) & 2005 \\
\hline & & $\begin{array}{l}\text { Katholieke Universiteit Leuven/Bélgica (2) } \\
\text { Instituto de Ciências Biomédicas da UFRJ (1) }\end{array}$ & 2006 \\
\hline & & $\begin{array}{l}\text { Katholieke Universiteit Leuven/Bélgica (2) } \\
\text { Instituto de Ciências Biomédicas da UFRJ (1) }\end{array}$ & 2006 \\
\hline & & $\begin{array}{l}\text { Instituto de Biociências da UFRGS (1) } \\
\text { Instituto de Ciências Biológicas da UFG (2) }\end{array}$ & 2006 \\
\hline & & & tinua... \\
\hline
\end{tabular}

Enc. Bibli: R. Eletr. Bibliotecon. Ci. Inf., Florianópolis, n. 26, 2ºm.2008 


\begin{tabular}{|c|c|c|c|}
\hline \multicolumn{2}{|c|}{ FREQÜÊNCIA } & \multirow{2}{*}{ INSTITUIÇÃO } & \multirow{2}{*}{ ANO } \\
\hline AUTOR & ARTIGOS & & \\
\hline \multicolumn{4}{|c|}{ Contitunação. } \\
\hline \multirow{10}{*}{2} & \multirow{10}{*}{10} & $\begin{array}{l}\text { UFRJ (1) } \\
\text { CNPq/DF (1) }\end{array}$ & 1995 \\
\hline & & Instituto de Bioquímica Médica da UFRJ (2) & 1996 \\
\hline & & $\begin{array}{l}\text { UFF (1) } \\
\text { UFRJ (1) }\end{array}$ & 1998 \\
\hline & & Instituto de Bioquímica Médica da UFRJ (2) & 1998 \\
\hline & & Instituto Dante Pazzanese de Cardiologia de São Paulo (2) & 1998 \\
\hline & & Instituto Dante Pazzanese de Cardiologia de São Paulo (2) & 1999 \\
\hline & & Instituto de Bioquímica Médica da UFRJ (2) & 2001 \\
\hline & & Pró-Reitoria de Pesquisa da USP (2) & 2002 \\
\hline & & $\begin{array}{l}\text { Instituto de Ciências Biomédicas da UFRJ (1) } \\
\text { University Department of Information Science/Inglaterra (1) }\end{array}$ & 2003 \\
\hline & & $\begin{array}{l}\text { INPI (1) } \\
\text { Instituto de Ciências Biomédicas da UFRJ (1) }\end{array}$ & 2004 \\
\hline
\end{tabular}

Quadro 2 - Afiliação institucional das autorias compartilhadas

Um dos artigos, publicado em 2003, possui cinco autores que se apresentam ligados institucionalmente a USP. Quatro autores compartilharam a autoria de quatro artigos, sendo a metade publicada na década de 1990. Eles se apresentam como investigadores do Instituto de Ciências Biológicas da UFRJ, Departamento de Bioquímica da UFRGS, Instituto de Ciências Biomédicas da UFRJ, Departamento de Psiquiatria e Medicina Legal da UFRJ e da Faculdade de Filosofia, Ciências e Letras de Ribeirão Preto da USP. Neste caso não houve cooperação institucional internacional para publicação.

Dos dez artigos com três autores, oito deles publicados após 2000, e estão distribuídos entre pesquisadores da Embrapa, Departamento de Estatística da Universidade de Brasília (UnB), Instituto Dante Pazzanese de Cardiologia de São Paulo, Instituto de Saúde de São Paulo, Faculdade de Economia, Administração e Contabilidade da USP, Faculdade de Medicina da UFRJ, Instituto de Ciências Biomédicas da UFRJ, Instituto de Química da USP, Instituto de Ciências Biológicas da Universidade Federal de Goiás (UFG) e Instituto de Biociências da UFRGS. Foram publicados dois artigos em co-autoria com um investigador da Katholieke Universiteit Leuven da Bélgica ambos publicados em 2006.

São também dez os artigos com dois autores, a maioria publicados na década de 1990, e estão distribuídos entre pesquisadores brasileiros do Instituto de Bioquímica Médica da Universidade Federal do Rio de Janeiro (UFRJ), CNPq, Universidade Federal Fluminense (UFF), Instituto Dante Pazzanese de Cardiologia de São Paulo, Pró-Reitoria de Pesquisa da USP e Instituto Nacional de Propriedade Industrial (INPI). Somente um dos artigos é compartilhado com um pesquisador da University Department of Information Science da Inglaterra publicado em 2003.

De todas as instituições a UFRJ foi a que mais compartilhou autorias e também a de mais presença na Scientometrics, conforme se observa comparando o Quadro 2 e a Tabela 2: 
Tabela 2: Presença das Instituições nas autorias

\begin{tabular}{|c|c|c|}
\hline INSTITUIÇÃO & FREQÜÊNCIA & $(\%)$ \\
\hline Instituto de Ciências Biomédicas da UFRJ & 10 & 14,08 \\
\hline Instituto de Bioquímica Médica da UFRJ & 7 & 9,86 \\
\hline Instituto Dante Pazzanese de Cardiologia de São Paulo & 6 & 8,45 \\
\hline Instituto de Ciências Biológicas da UFG & 5 & 7,04 \\
\hline Departamento de Bioquímica da UFRGS & 4 & 5,63 \\
\hline Embrapa & 4 & 5,63 \\
\hline Faculdade de Filosofia, Ciências e Letras de Ribeirão Preto da USP & 4 & 5,63 \\
\hline Instituto de Ciências Biológicas da UFRJ & 4 & 5,63 \\
\hline Katholieke Universiteit Leuven/Bélgica & 4 & 5,63 \\
\hline Departamento de Estatística da UnB & 2 & 2,82 \\
\hline Departamento de Psiquiatria e Medicina Legal UFRJ & 2 & 2,82 \\
\hline Faculdade de Medicina UFRJ & 2 & 2,82 \\
\hline Núcleo de Pesquisa em Relações Internacionais & 2 & 2,82 \\
\hline Núcleo de Pesquisas sobre Ensino Superior & 2 & 2,82 \\
\hline Pró-Reitoria de Pesquisa da USP & 2 & 2,82 \\
\hline UFRJ & 2 & 2,82 \\
\hline $\mathrm{CNPq} / \mathrm{DF}$ & 1 & 1,41 \\
\hline Faculdade de Economia, Administração e Contabilidade da USP & 1 & 1,41 \\
\hline INPI & 1 & 1,41 \\
\hline Instituto de Biociências da UFRGS & 1 & 1,41 \\
\hline Instituto de Matemática e Estatística USP & 1 & 1,41 \\
\hline Instituto de Química da USP & 1 & 1,41 \\
\hline Instituto de Saúde de São Paulo & 1 & 1,41 \\
\hline UFF & 1 & 1,41 \\
\hline University Department of Information Science/Inglaterra & 1 & 1,41 \\
\hline TOTAL & 71 & 100 \\
\hline
\end{tabular}

A UFRJ compartilhou autorias com as seguintes instituições: UFRGS, Instituto Dante Pazzanese de Cardiologia de São Paulo, CNPq, UFF, INPI e as internacionais Katholieke Universiteit Leuven da Bélgica e University Department of Information Science da Inglaterra. Neste caso, a responsabilidade coube ao Instituto de Ciências Biomédicas da UFRJ, provavelmente com os mesmos autores, como se poderá ver adiante. As demais autorias compartilhadas o foram com instituições brasileiras. Há momentos de parcerias e outros em que a autoria é única em todas as instituições apresentadas. A própria UFRJ apresentou-se sem compartilhamento em seis artigos.

Para identificar os autores mais produtivos, examinaram-se os 47 autores envolvidos na produção dos 34 artigos publicados na Scientometrics de 1978 até 2006. Destes, 44 autores são brasileiros e estão arrolados na Tabela 3:

Tabela 3 - Autores brasileiros dos artigos da amostra

\begin{tabular}{clrr}
\hline NÚMERO & AUTOR & FREQÜENEIA DE ARTIGOS & $(\%)$ \\
\hline $\mathbf{1}$ & Leta, J. & $\mathbf{9}$ \\
$\mathbf{2}$ & De Meis, L. & $\mathbf{1 2 , 0}$ \\
$\mathbf{3}$ & Pereira, J. C. R. & $\mathbf{6 , 7}$ \\
$\mathbf{4}$ & Escuder, M. M. L. & $\mathbf{4}$ & $\mathbf{5 , 3}$ \\
$\mathbf{5}$ & Velloso, S. & $\mathbf{3}$ & $\mathbf{4 , 0}$ \\
6 & Bini, L. M. & $\mathbf{3}$ & $\mathbf{4 , 0}$ \\
7 & Carvalho, P. & 2 & 2,7 \\
& E. & 2,7
\end{tabular}

Enc. Bibli: R. Eletr. Bibliotecon. Ci. Inf., Florianópolis, n. 26, 2ºm.2008 


\begin{tabular}{|c|c|c|c|}
\hline NÚMERO & AUTOR & FREQÜÊNCIA DE ARTIGOS & $(\%)$ \\
\hline 8 & Chaimovich, H. & 2 & 2,7 \\
\hline 9 & Figueira, I. & 2 & 2,7 \\
\hline 10 & Fonseca, L. & 2 & 2,7 \\
\hline 11 & Jacques, R. & 2 & 2,7 \\
\hline 12 & Lannes, D. & 2 & 2,7 \\
\hline 13 & Meneghini, R. & 2 & 2,7 \\
\hline 14 & Souza, G. S. & 2 & 2,7 \\
\hline 15 & Velho, L. & 2 & 2,7 \\
\hline 16 & Wofchuk, S. & 2 & 2,7 \\
\hline 17 & Zanotto, E. D. & 2 & 2,7 \\
\hline 18 & Alves, E. & 1 & 1,3 \\
\hline 19 & Ávila, A. F. D. & 1 & 1,3 \\
\hline 20 & Balbachevsky, E. & 1 & 1,3 \\
\hline 21 & Batista, P. D. & 1 & 1,3 \\
\hline 22 & Cabral, J. E. D. & 1 & 1,3 \\
\hline 23 & Campiteli, M. G. & 1 & 1,3 \\
\hline 24 & Christovão, H. T. & 1 & 1,3 \\
\hline 25 & Coutinho, M. & 1 & 1,3 \\
\hline 26 & Cruz, E. R. & 1 & 1,3 \\
\hline 27 & Diniz-Filho, J. A. F. & 1 & 1,3 \\
\hline 28 & Dos Santos, N. F. & 1 & 1,3 \\
\hline 29 & Fischer, A. L. & 1 & 1,3 \\
\hline 30 & Friedrich, M. P. & 1 & 1,3 \\
\hline 31 & Guimarães, J. A. & 1 & 1,3 \\
\hline 32 & Holzhacker, D. N. & 1 & 1,3 \\
\hline 33 & Humann, M. C. & 1 & 1,3 \\
\hline 34 & Kinouchi, O. & 1 & 1,3 \\
\hline 35 & Martinez AS & 1 & 1,3 \\
\hline 36 & Melo, A. S. & 1 & 1,3 \\
\hline 37 & Nunes, E. D. & 1 & 1,3 \\
\hline 38 & Oliveira, P. L. & 1 & 1,3 \\
\hline 39 & Patrão, D. da C. & 1 & 1,3 \\
\hline 40 & Pinheiro-Machado, R. & 1 & 1,3 \\
\hline 41 & Quirino, T. R. & 1 & 1,3 \\
\hline 42 & Rodrigues, P. D. & 1 & 1,3 \\
\hline 43 & Rumjanek, V. M. & 1 & 1,3 \\
\hline 44 & Vencio, R. N. Z. & 1 & 1,3 \\
\hline TOTAL & & 75 & 100 \\
\hline
\end{tabular}

São cinco os autores mais produtivos: Leta da UFRJ, com nove artigos (12\%), seguido por De Meis da UFRJ com cinco artigos (6,7\%) e Pereira do Instituto Dante Pazzanese de Cardiologia de São Paulo, com quatro artigos (5,3\%). Escuder do Instituto Dante Pazzanese de Cardiologia de São Paulo e Velloso da UFRGS publicaram três artigos (4\%) cada um. Todos estes autores estão ligados às ciências biológicas e da saúde. Depois se encontra 12 autores com dois artigos cada um e 27 autores com um artigo cada um.

Conforme descrito na Tabela 4, são três os autores estrangeiros que colaboraram na produção de cinco artigos, dois deles, Glanzel e Thijs pesquisadores ligados a instituições belgas do campo das ciências biológicas e da saúde e Lewison investigador inglês subordinado a uma instituição do campo da Ciência da Informação. 
Tabela 4 - Autores estrangeiros dos artigos da amostra

\begin{tabular}{llcc}
\hline NÚMERO & AUTOR & PAÍS & FREQÜÊNCIA DE ARTIGOS \\
\hline 1 & Glanzel, W. & Bélgica & 2 \\
2 & Thijs, B. & Bélgica & 2 \\
3 & Lewison, G. & Inglaterra & 1 \\
\hline TOTAL & & & 5 \\
\hline
\end{tabular}

Apesar de o recorte temporal iniciar-se com a primeira edição da revista Scientometrics em 1978, oito anos após a criação do primeiro curso de Mestrado em CI no Brasil, encontra-se o primeiro artigo publicado por pesquisadores brasileiros que utilizaram a bibliometria como método ou teoria somente em 1985. A evolução das publicações conforme definido nesta pesquisa, podem ser visualizadas no Gráfico 1. Os anos que apresentaram o maior número de publicações, cinco ou 14,71\% da amostra, foram 1998 e 2006.

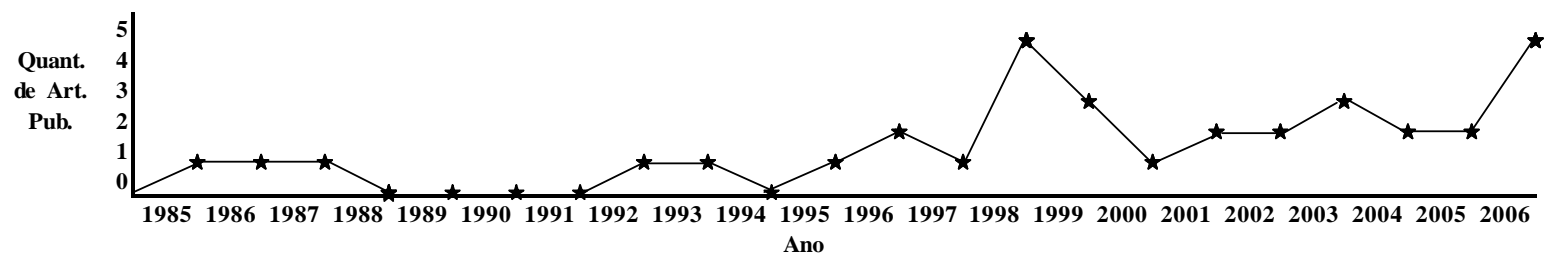

Gráfico 1 - Ano de publicação dos artigos da amostra

Percebe-se baixa freqüência de artigos de autoria de pesquisadores brasileiros na revista Scientometrics. De 1985 até 2006, em 22 anos de publicação, nossos pesquisadores contribuíram com 34 artigos, com uma média de 1,5\% textos por ano. Este resultado é corroborado pelo estudo de Dutt, Carg e Bali (2003) que analisaram 1.317 papers publicados no periódico Scientometrics de 1978 até 2001 e neste período o Brasil contribuiu com 19 artigos, ou 1,44\% da amostra daquela investigação.

Desta amostra apenas cinco artigos não receberam citação e foram publicados em 1996, em 1998 e três em 2006, ou seja, ainda com possibilidade de receber citações. Encontrou-se cinco artigos que obtiveram 10,11, 13, 18 e 25 citações, representando uma boa repercussão dos artigos produzidos por autores brasileiros, são eles:

10 citações para: LETA, J.; LANNES, D.; De MEIS, L. Human resources and scientific productivity in Brazil. Scientometrics, v. 41, n. 3, p. 313324, Mar./Apr. 1998.

11 citações para: MENEGHINI, R. Brazilian production in biochemistry: the question of international versus domestic publication. Scientometrics, v. 23, n. 1, p. 21-30, Jan. 1992.

13 citações para: MENEGHINI, R. The key role of collaborative work in the growth of Brazilian science in the last ten years. Scientometrics, v. 35, n. 3, p. 367-373, Mar./Apr. 1996.

18 citações para: LETA, J.; De MEIS, L. A profile of science in Brazil. Scientometrics, v. 35, n. 1, p. 33-44, Jan. 1996.

25 citações para: VELHO, L. The meaning of citation in the context of a scientifically peripheral country. Scientometrics, v. 9, n. 1-2, p. 71-89, Jan. 1986.

Buscando visualizar o número de vezes que os artigos eleitos nesta amostra foram Enc. Bibli: R. Eletr. Bibliotecon. Ci. Inf., Florianópolis, n. 26, 2ºm.2008 
citados por outros autores resumimos os dados na Tabela 5:

Tabela 5 - Número de citações que os artigos da amostra receberam

\begin{tabular}{lcc}
\hline FREQÜÊNCIA DE ARTIGOS & FREQÜÊNCIA DE CITAÇÕES RECEBIDAS \\
\hline 1 & 25 \\
1 & 18 \\
1 & 13 \\
1 & 11 \\
1 & 10 \\
5 & 6 \\
2 & 5 \\
1 & 4 \\
3 & 3 \\
6 & 2 \\
7 & 1 \\
& 5 & 0 \\
\hline TOTAL & $\mathbf{3 4}$ & $\mathbf{9 8}$ \\
\hline
\end{tabular}

\section{CONSIDERAÇÕES FINAIS}

Atendendo ao objetivo desta análise identificaram-se os pesquisadores brasileiros que investigaram e publicaram sobre bibliometria no periódico internacional especializado na área, Scientometrics de 1978 até 2006, especificando por:

a) o tipo de autoria dos artigos - única ou compartilhada: do total de 34 artigos recuperados nove apresentaram autoria única (26,48\%). Percebeu-se, portanto, uma preferência pela autoria compartilhada, distribuídos na seguinte proporção: um artigo com cinco autores (2,94\%), quatro artigos com quatro autores (11, 76\%) e 20 artigos com dois e três autores $(29,41 \%)$ da amostra cada um, num total de 25 artigos de autoria compartilhada ou múltipla (73,52\%). Ao contrário o estudo de Machado (2007) que verificou o predomínio de autoria única na sua amostra de 21 artigos em bibliometria nos periódicos nacionais, esse estudo verificou a preferência pela autoria compartilhada.

b) a cooperação institucional para publicação dos artigos: dos 25 artigos com autoria múltipla a maioria divide com autores nacionais, exceto em três casos em que compartilham com pesquisadores da Inglaterra (1) e da Bélgica (2). O único artigo com cinco autores é compartilhado em nível interno com pesquisadores da USP de diferentes núcleos ou departamentos de pesquisas.

c) a afiliação institucional dos autores apresenta uma multiplicidade de atores, mas, predominantemente pertencentes a academias e institutos públicos, exceto o Instituto Dante Pazzanese, da Fundação Adib Jatene, assim distribuídos em ordem crescente de colaborações: UFRJ; USP; Instituto Dante Pazzanese de Cardiologia de São Paulo; UFRGS; Embrapa, UFG e Katholieke Universiteit Leuven da Bélgica, UnB, CNPQ, UFF, INPI e University Department of Information Science da Inglaterra.

d) os autores mais produtivos: dos 47 autores envolvidos na produção dos 34 artigos publicados no Scientometrics são Jacqueline Leta da UFRJ, com nove artigos (12\%), seguido por Leopoldo De Meis da UFRJ com cinco artigos (6,7\%) e J. C. R Pereira, do Instituto Dante Pazzanese de Cardiologia de São Paulo, com quatro artigos (5,3\%). M. M. L. Escuder, do Enc. Bibli: R. Eletr. Bibliotecon. Ci. Inf., Florianópolis, n. 26, $2^{\circ}$ sem.2008 
Instituto Dante Pazzanese de Cardiologia de São Paulo e S.Velloso da UFRGS que publicaram três artigos (4\%) cada um. Todos estes autores estão ligados às ciências biológicas e da saúde. Encontraram-se doze autores com dois artigos cada um e 27 autores com apenas um artigo. Destacam-se os cinco mais produtivos:

Jacqueline Leta: formada em Ciências Biológicas com mestrado e doutorado em Química Biológica e Gestão, Educação e Difusão em Ciências. É professora do Instituto de Ciências Biológicas da UFRJ, assessora do MCT e pesquisadora junto à USP e FAPESP. Desenvolve trabalhos com pesquisadores de outros países, acentuadamente da Bélgica e da Inglaterra. Foi orientanda de Leopoldo de Meis, segundo autor mais produtivo, deste trabalho, na especialização, mestrado e doutorado.

Leopoldo de Meis: formou-se médico e atualmente é professor de bioquímica do Instituto de Bioquímica Médica da UFRJ, reconhecido internacionalmente e detentor de vários prêmios científicos no Brasil e exterior, tendo orientado mais de 30 teses de doutorado.

Julio César Rodrigues Pereira: concluiu o doutorado em Epidemiologia pela Faculdade de Saúde Pública da USP em 1995 e Docência Livre em 2004. É médico pela Escola Paulista de Medicina (1977) e Master of Sciences in Medical Sciences pela Universidade de Bristol - UK em 1989. Atualmente é Professor Associado da USP e Professor de Pós-Graduação. É líder do Grupo de Pesquisa do Laboratório de Epidemiologia e Estatística do Instituto Dante Pazzanese de Cardiologia.

Maria Mercedes Loureiro Escuder: possui mestrado em Epidemiologia e Estatística pela Faculdade de Saúde Pública da Universidade de São Paulo (1998). Atualmente é pesquisadora científica do Instituto de Saúde, com sede em São Paulo. Participa do Grupo de Pesquisa do Laboratório de Epidemiologia e Estatística juntamente com Júlio César Rodrigues Pereira. Sobre a pesquisadora Sancia Velloso só foi possível conseguir a informação que a pesquisadora: pertencia ao Instituto de Ciências Biomédicas da UFRJ.

e) o ano da publicação dos artigos: O primeiro artigo publicado por autores brasileiros na Scientometrics foi em 1985, quinze anos depois da criação do primeiro curso de Mestrado em CI no Brasil. Os anos de 1998 e 2006 apresentaram o maior número de publicações.

f) freqüência em que os artigos foram citados por outros autores: apenas cinco artigos não receberam citação e foram publicados em 1996, 1998 e três em 2006. Estes últimos ainda com possibilidade de receber citações. Cinco artigos obtiveram entre 10 e 25 citações, representando uma boa repercussão.

A análise permite concluir que não há pesquisadores cientistas da informação entre os autores brasileiros mais produtivos que publicaram na revista Scientometrics de 1978 até 2006. Dos autores que receberam mais citações, há uma autora que tem sua produção relacionada à CI, Lea Velho, ligada institucionalmente ao CNPq cuja formação é em engenharia agronômica, professora da UNICAMP e que desenvolve há anos pesquisas cientométricas.

Constatou-se, também, uma baixa cooperação institucional internacional para publicação e a participação de pesquisadores brasileiros junto a esta publicação como escassa: segundo o SSCI (2007) foram publicados 2.187 artigos na revista de 1978 até 2006, ou seja, os 34 artigos de autores brasileiros publicados representam 1,55\% do total de artigos.

Estes resultados podem funcionar como um alerta para a necessidade de serem fortalecidos os laços entre a CI do Brasil, na área de bibliometria e cienciometria, com os pesquisadores de outros países. Esperava-se, ao iniciar a pesquisa, encontrar maior produção neste sentido, mas não ocorreu. A expectativa se baseava na suposição de que os cursos de pós-graduação realizados no exterior possibilitariam a continuidade das relações com os pesquisadores. Se isso aconteceu, não foi em forma de publicações de artigos na Scientometrics.

Enc. Bibli: R. Eletr. Bibliotecon. Ci. Inf., Florianópolis, n. 26, $2^{\circ}$ sem.2008 
Para que pesquisas bibliométricas sejam mais desenvolvidas seria importante trabalhar estes temas nos ensinos de graduação para despertar o interesse de pesquisadores em potencial, possivelmente existentes nas escolas de biblioteconomia e cursos afins. Apontam-se como alternativas para agregar os pesquisadores da área e fortalecer o compartilhamento de estudos, a realização de eventos específicos em bibliometria no país e possibilitando a participação de eventos no exterior.

Concluindo ficam aqui algumas reflexões: apesar do primeiro Mestrado em CI ter introduzido a Bibliometria no Brasil (URBIZAGÁSTEGUI ALVARADO, 1984), porque a produção científica neste importante periódico internacional é mais destacada entre pesquisadores brasileiros de outras áreas que não a CI? Seria pelo tímido relacionamento dos pesquisadores brasileiros do campo com pesquisadores extra-muros? Ou seria pelo não domínio da língua inglesa? Eventualmente o idioma pode se tornar um obstáculo. Meadows (1999) esclarece que de acordo com o campo de pesquisa exige-se que os autores para publicarem, escrevam normalmente em língua inglesa.

Ou ainda, haveria pouco interesse dos pesquisadores em CI na Bibliometria? Seria comparável a produção sobre o tema em periódicos nacionais e internacionais? Población e Noronha (2002) em seu artigo sobre a produção dos docentes/doutores dos programas de pósgraduação em CI no Brasil de 1990 até 1999 apontam que 66 docentes tiveram a produção global de 1.108 documentos, sendo $37,3 \%$ ou 414 documentos produzidos na tipologia artigos de periódicos. Infelizmente, apesar deste tipo de documento ter sido o mais representativo da amostra, as autoras não identificam no texto os títulos dos periódicos onde os artigos foram publicados pelos pesquisadores ou o local de publicação dos mesmos, informações que poderiam indicar uma visibilidade internacional.

Os indicadores apontados neste estudo apresentam um quadro do desenvolvimento da bibliometria produzida pelos pesquisadores brasileiros e sua visibilidade internacional num periódico específico. No entanto, não esgota a possibilidade de novos estudos, incluindo outras fontes de informação a exemplo do que fizeram os autores preocupados com o tema e aqui citados e que poderão apresentar outros tantos vieses da bibliometria no Brasil.

\section{REFERÊNCIAS}

AIMS AND Scope. In: Scientometrics. New York: Springer, 1978- . Disponível em: http://www.springer.com/computer/database+management+\%26+information+retrieval/journ al/11192?detailsPage=aimsAndScopes. Acesso em: 13 jul. 2008.

ARENAS, J. L. et al. Una visión bibliométrica de la investigación en bibliotecología y ciencia de la información de América Latina y el Caribe. Revista Española de Documentación Científica, v. 23, n. 1, p. 45-62, Enero/marzo 2000.

CARG, K. C. An overview of cross-national, national, and institutional assessment as reflected in the international journal Scientometrics. Scientometrics, v. 56, n. 2, p. 169-199. 2003. Disponível em: http://www.springerlink.com/content/k93q452q51q18227/fulltext.pdf. Acesso em: 09 jul. 2008.

CASTRO, Regina C. Figueiredo. Revistas de cirurgia e gastroenterologia: indexação em bases de dados e indicadores bibliométricos. Acta Cirúrgica Brasileira, São Paulo, v. 21, n. 3, 2006. Disponível em: < http://www.scielo.br/scielo.php?script=sci_arttext\&pid=S010286502006000300002\&lng=pt\&nrm=iso. Acesso em: 08 mar 2008. 
DUTT, B.; CARG, K. C.; BALI, A. Scientometrics of the international journal Scientometrics. Scientometrics, v. 56, n. 1, p. 81-93. 2003. Disponível em: http://www.springerlink.com/content/g40818211336p233/fulltext.pdf. Acesso em: 09 jul. 2008.

HERRERO-SOLANA, V.; RÍOS-GÓMEZ, C. Producción latinoamericana en biblioteconomía y documentación en el Social Science Citation Index (SSCI) 1966-2003. Information Research, v. 11, n. 2, January, 2006. Disponível em: http://informationr.net/ir/11-2/paper247.html. Acesso em 9 jul. 2007.

MACHADO, Raymundo das Neves. Análise cientométrica dos estudos bibliométricos publicados em periódicos da área de biblioteconomia e ciência da informação (1990-2005). Perspectivas em Ciência da Informação, Belo Horizonte, v.13, n.3, p. 2-20, 2007.

MACIAS-CHAPULA, C. A. O papel da informetria e da cienciometria e sua perspectiva nacional e internacional. Ciência da Informação, Brasília, DF, v. 27, n. 2, p. 134-140, maio/ago. 1998.

MEADOWS, A. J. A comunicação científica. Brasília, DF: Briquet de Lemos, 1999.

MOYA-ANEGÓN, F.; HERRERO-SOLANA, V. Visibilidad internacional de la producción científica iberoamericana en biblioteconomía y documentación (1991-2000). Ciência da Informação, Brasília, v. 31, n. 3, p. 54-65, set./dez. 2002.

MUGNAINI, R.; JANNUZZI, P. M.; QUONIAM, L. Indicadores bibliométricos da produção científica brasileira: uma análise a partir da base Pascal. Ciência da Informação, Brasília, v. 33, n. 2, 2004. Disponível em:http://www.scielo.br/scielo.php?script=sci_arttext\&pid=S010019652004000200013\&lng=pt\&nrm=iso. Acesso em: 13 jul. 2008.

PACKER, A. L.; MENEGHINI, R. Visibilidade da produção científica. In: POBLACIÓN, D. A.; WITTER, G. P.; SILVA, J. F. M. (Orgs.). Comunicação e produção científica: contexto, indicadores, avaliação. São Paulo: Angellar, 2006. p. 235-260.

POBLACIÓN, D. A.; NORONHA, D. P. Produção das literaturas branca e cinzenta pelos docentes/doutores dos programas de pós-graduação em Ciência da Informação no Brasil. Ciência da Informação, Brasília, v. 31, n. 2, p. 98-106, maio/ago. 2002. Disponível em: http://www.scielo.br/pdf/ci/v31n2/12913.pdf. Acesso em: 13 jul. 2008.

SENGUPTA, I. N. Bibliometrics, informetrics, scientometrics and librametrics: an overview. Libri, Copenhagen, v. 42, n. 2, p. 75-98, 1992.

STUMPF, I. R. C. et al. Usos dos termos cienciometria e cientometria pela comunidade científica brasileira. In: POBLACIÓN, D. A.; WITTER, G. P.; SILVA, J. F. M. (Org.). Comunicação e produção científica: contexto, indicadores, avaliação. São Paulo: Angellar, 2006. p. 341-370.

TESTA, J. A base de dados ISI e seu processo de seleção de revistas. Ciência da Informação, Brasília, v. 27, n. 2, p. 233-235, maio/ago. 1998. 
THOMSON CORPORATION. Web of Science: SO=(SCIENTOMETRICS) DocType=All document types; Language=All languages; Database=SSCI; Timespan=1978-2006. 2007. Disponível em: http://portal.isiknowledge.com/portal.cgi?DestApp=WOS\&Func=Frame. Acesso em 30 jul. 2007.

URBIZAGÁstegUi ALVARADO, R. A bibliometria no Brasil. Ciência da Informação, Brasília, DF, v. 13, n. 2, p. 91-105, jul./dez. 1984.

VANTI, N. A. P. Da Bibliometria à webometria: uma exploração conceitual dos mecanismos utilizados para medir o registro e a difusão do conhecimento. Ciência da Informação, Brasília, v. 31, n. 2, p. 152-162, maio/ago. 2002.

\begin{abstract}
The aim this paper is to reveal the scientific production of Brazilian researchers who had their articles published in the journal Scientometrics from 1978 to 2006. The papers recovered were categorized by type of authorship, authors' affiliation and institutional cooperation, most productive authors, year of publication, and number of times the article was cited. The data analyzed reveal: (a) preference for shared authorship; (b) predominant institutional affiliation in universities and public institutes; (c) most productive authors were from the biological and health areas; (d) most articles were published in 1998 and 2006; and (e) five articles had from 10 to 25 citations. The research concluded that the participation the Brazilians researchers' in the publication is scarce, the international cooperation for publication is inexpressive, and it is possible conclude too the lack to researchers in the field of CI among the most productive authors.
\end{abstract}

KEYWORDS: Scientific production. Brazil. Journal. Scientometrics.

Originais recebidos em: 16/03/2008

Texto aprovado em: 02/09/2008 